\title{
A Single Amino Acid Substitution in the Virus-Encoded Replicase of Tomato Mosaic Tobamovirus Alters Host Specificity
}

\author{
Hiroshi Hamamoto, ${ }^{1}$ Yuichiro Watanabe, ${ }^{2}$ Hiroshi Kamada, ${ }^{1}$ and Yoshimi Okada ${ }^{2}$ \\ ${ }^{1}$ Gene Experiment Center, University of Tsukuba, Ten-noh Dai 1-1-1, Tsukuba, Ibaraki 305; ${ }^{2}$ Department of \\ Biosciences, Faculty of Science and Engineering, Teikyo University, Toyosatodai 1-1, Utsunomiya, To- \\ chigi 320, Japan \\ Accepted 16 July 1997.
}

Introduction of a single amino acid substitution (GIn979 to Ile) into the 130- and 180-kDa proteins of tomato mosaic tobamovirus (ToMV) rendered the mutant virtually unable to replicate in tomato cells while it replicated perfectly in tobacco cells. The mechanism of this change in host specificity is discussed.

Tomato mosaic tobamovirus (ToMV) has a genome of messenger-sense, single-stranded RNA of about 6,400 nucleotides. The RNA encodes three nonstructural proteins, of 130 $\mathrm{kDa}, 180 \mathrm{kDa}$ (the readthrough product of the $130-\mathrm{kDa}$ protein) and $30-\mathrm{kDa}$, respectively, and the coat protein (Ohno et al. 1984; Fig. 1). The genes for the $130-$ and $180-\mathrm{kDa}$ proteins exhibit homology to virus-encoded genes for replicases of other RNA viruses at the nucleotide level (Haseloff et al. 1984; Kamer and Argos 1984). These proteins are reported to be involved in viral replication (Ishikawa et al. 1986). Amino acid 979 of the 130- and $180-\mathrm{kDa}$ proteins of ToMV plays an important role in overcoming the tomato $\mathrm{Tm}-1$ resistance gene. The Tm- 1 gene inhibits replication of wild-type ToMV (Fraser and Loughlin 1980; Motoyoshi and Oshima 1979; Pelham 1972; Watanabe et al. 1987a), whereas a mutant ToMV, with a Gln979 to Glu substitution in the 130- and 180$\mathrm{kDa}$ proteins, replicates in $\mathrm{Tm}-1$ tomato cells (Meshi et al. 1988). In a previous study, we generated five mutants, with Gln979 to Asn, Asp, His, Lys, and Arg substitutions, respectively, in an attempt to elucidate the features of amino acid 979 that are important for overcoming the effect of the tomato Tm-1 resistance gene (Hamamoto et al. 1997). In this report, we describe the unusual behavior of another mutant that has a Gln979 to Ile substitution (TLIle).

Plasmid pTLIle was constructed by site-directed mutagenesis (Kunkel 1985) from pTLW3 (Hamamoto et al. 1993; Fig. 1), which has a $\mathrm{T} 7$ promoter for transcription of infectious

Corresponding author: H. Hamamoto; Fax: +81-298-53-6006. E-mail: hirohama@sakura.cc.tsukuba.ac.jp

Present address of Y. Watanabe: Department of Life Sciences, Graduate School of Arts and Sciences, University of Tokyo, Komaba 3-8-1, Meguro-ku, Tokyo 153, Japan. wild-type ToMV RNA in vitro. The oligonucleotide $5^{\prime}$ dCTTC CTAAATATTAGGTATGAAG 3' was used to introduce the mutation into the plasmid. Construction of the plasmid, transcription in vitro, and electroporation were performed as described previously (Hamamoto et al. 1997). In this report, the transcript from the plasmid is referred to in the same way as the plasmid but with deletion of the prefix " $p$ " from the designation of the plasmid. Tobacco BY-2 and tomato GCR 26 suspension-cultured cell lines were used for electroporation (Watanabe et al. 1987a, 1987b). When we inoculated these cells with wild-type ToMV transcript, about 40 to $70 \%$ of protoplasts were routinely infected. For plant-inoculation experiments, we used 5- to 6-week-old tobacco (Nicotiana tabacum cv. Samsun) plants and 4- to 5-week-old tomato (Lycopersicon esculentum cv. Craigella GCR $26[+/+]$; Pelham 1972) plants.

We inoculated tobacco and tomato protoplasts with TLW3 and TLIle by electroporation to examine the replication of TLIle in single cells. Total RNA and proteins were extracted after an 18-h incubation and analyzed as described previously (Hamamoto et al. 1997). To confirm that similar levels of RNAs were analyzed, blots of rRNA were carried out (data not shown). In tobacco BY-2 protoplasts, TLW3 and TLIle RNA and coat protein accumulated to similar levels. However, in GCR 26 protoplasts, we failed to detect any accumulation of RNA or coat protein in TLIle- inoculated protoplasts, while

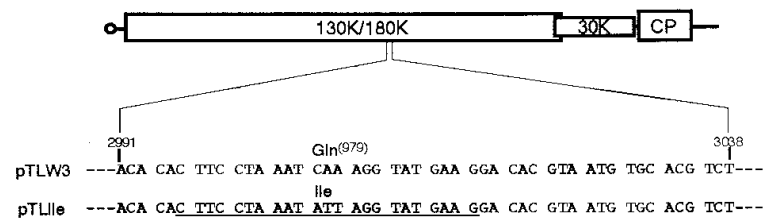

Fig. 1. Schematic representation of the genomic RNA of tobacco mosaic tobamovirus and sequences between nucleotides 2991 and 3038 of plasmids used in this study. Coding regions for the 130- and $180-\mathrm{kDa}$ $(130 \mathrm{~K} / 180 \mathrm{~K}), 30-\mathrm{kDa}(30 \mathrm{~K})$, and coat $(\mathrm{CP})$ proteins are represented by open boxes, noncoding regions by lines. An open circle on the left indicates a cap structure. Underlined nucleotides indicate the sequence of the oligonucleotide that was synthesized for in vitro mutagenesis of plasmids. Amino acid 979 is indicated above the nucleotide sequence. 
TLW3-inoculated protoplasts accumulated both RNA and coat protein (Fig. 2). These results indicate that a single amino acid substitution (Gln979 to Ile) rendered ToMV unable to detectably replicate in tomato cells.

Tobacco and tomato plants were inoculated with TLW3 and TLIle. Each transcript was combined with TMV-OM coat protein for reconstitution of viral particles, as described previously (Meshi et al. 1986), and used for inoculation.

In tobacco plants, both TLW3 and TLIle caused systemic

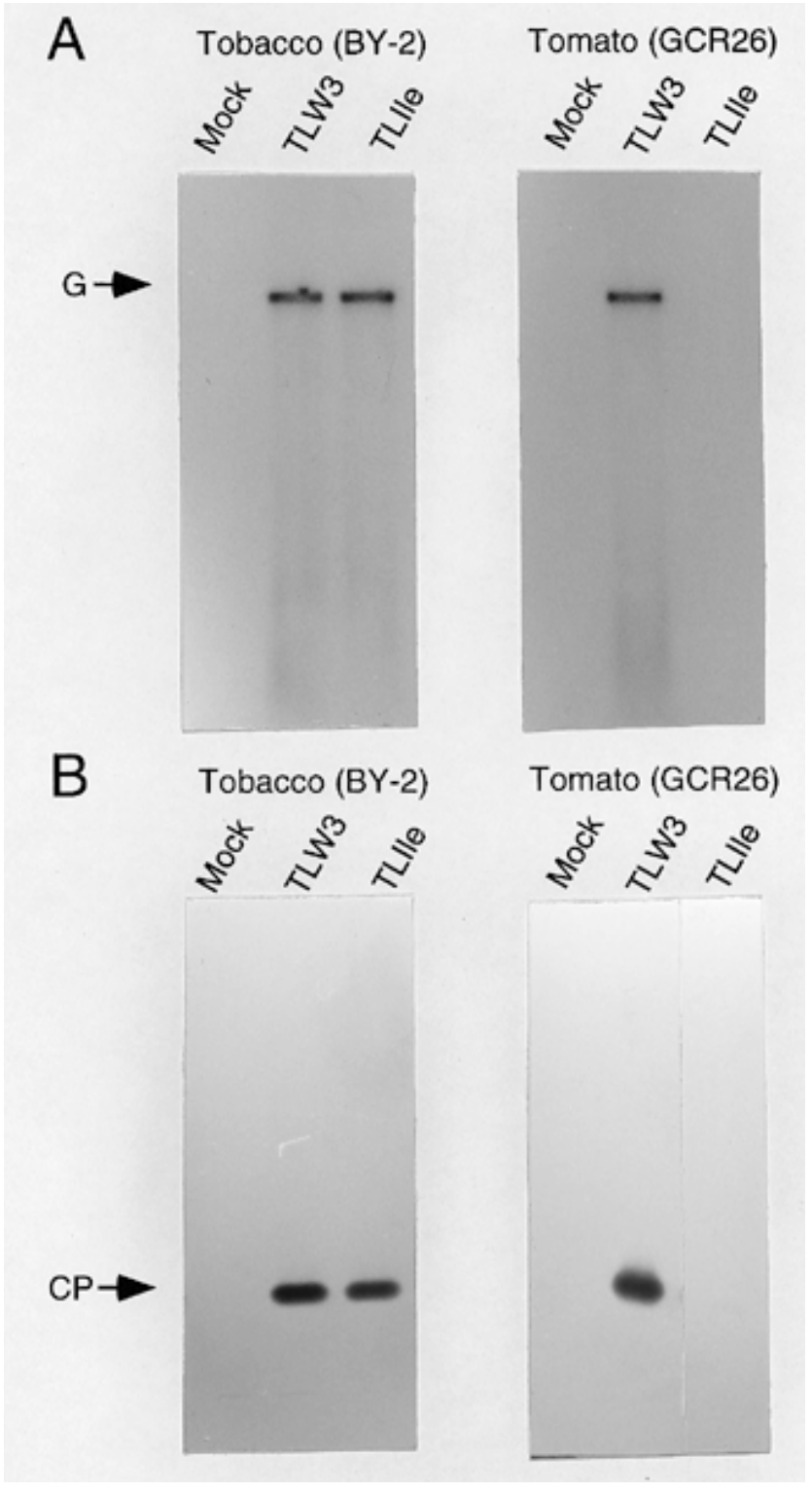

Fig. 2. Accumulation of TLW3 and TLIle RNAs and production of coat protein in tobacco BY-2 and tomato GCR 26 protoplasts. A, Northern (RNA) blotting analysis of the accumulated RNA $18 \mathrm{~h}$ after introduction of TLW3 and TLIle into tobacco BY-2 and tomato GCR 26 protoplasts by electroporation. Total RNA extracted from $5 \times 10^{4}$ protoplasts was analyzed. pTLW3 was used as the probe. Position of the genomic RNA of tobacco mosaic tobamovirus (ToMV) is indicated (G). B, Immunoblotting analysis of accumulated coat protein $18 \mathrm{~h}$ after electroporation of tobacco BY-2 and tomato GCR 26 protoplasts. Antiserum raised in rabbit against ToMV was used for immunostaining. Total protein extracted from $5 \times 10^{3}$ protoplasts was analyzed. Position of the coat protein $(\mathrm{CP})$ is indicated. mosaic symptoms and coat protein was detected in the upper leaves 14 days after inoculation (data not shown). The progeny viruses of both transcripts were harvested from the upper leaves as described previously (Otsuki et al. 1977). The yields of virus particles were 0.5 to $1 \mathrm{mg}$ per $\mathrm{g}$ of fresh leaves. The sequence around the mutagenized area of the viral genome was examined by reverse transcription-polymerase chain reactions (RT-PCR) as described previously (Hamamoto et al. 1997). No secondary mutations were detected within the sequence that was clearly read (nucleotides 2956 to 3336).

In tomato plants, in one experiment (E1), mosaic symptoms were observed in TLW3-inoculated plants while TLIleinoculated plants remained symptomless 14 days after inoculation. Total protein was extracted from the upper noninoculated leaves and analyzed. Unexpectedly, we detected the coat protein not only in TLW3-inoculated plants but also in two (E1-P1 and E1-P2) of five plants that had been inoculated with TLIle (data not shown). In another experiment (E2), total protein was extracted from upper noninoculated leaves 4,7 , 10 , and 14 days after inoculation and analyzed. In the case of TLW3-inoculated plants, ToMV coat protein was detected in all four plants 4 days after inoculation. In the case of TLIleinoculated tomato plants, coat protein was detected in only one of five plants (E2-P2) after 7 days and in three plants (E2P1, E2-P2, and E2-P5) after 10 days (Fig. 3).

We postulated that the delayed propagation of TLIle might have been due to occurrence of mutants that could replicate in tomato cells. Therefore, we analyzed the sequence around the codon for amino acid 979 in the genome of the progeny virus.

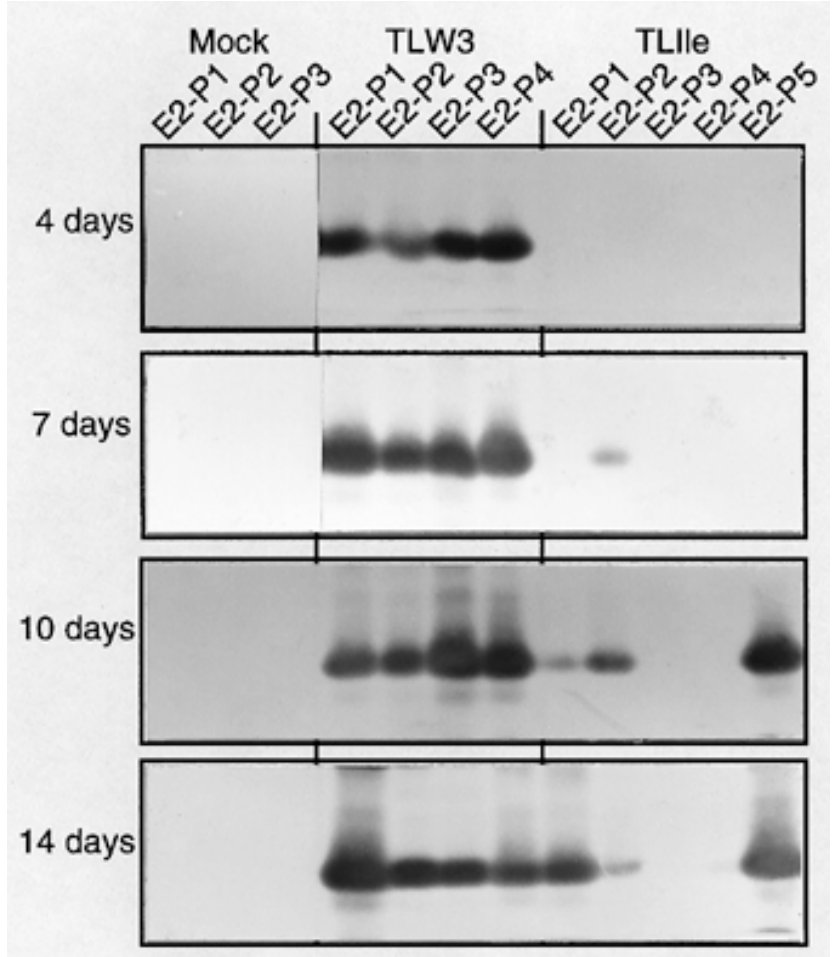

Fig. 3. Immunoblotting analysis of proteins extracted from upper noninoculated leaves of tomato GCR 26 plants that had been mock-inoculated or inoculated with TLW3 or TLIle in E2 experiment. Proteins were extracted $4,7,10$, and 14 days after inoculation. Total proteins extracted from $0.02 \mathrm{mg}$ of leaf tissue were analyzed. Antiserum raised in rabbit against tobacco mosaic tobamovirus was used for immunostaining. 
We extracted total RNA from the upper leaves of each plant 14 days after inoculation and subjected it to RT-PCR. RT-PCR and construction of an M13 phage library of the SalI-BamHI fragment (nucleotides 2941 to 3336) of the product of RTPCR from the progeny virus were carried out as described previously (Hamamoto et al. 1997). From the library of the progenies of two TLW3-inoculated plants (E2-P1 and E2-P2) and five TLIle-inoculated plants (E1-P1, E1-P2, E2-P1, E2$\mathrm{P} 2$, and E2-P5), at least five M13-clones were picked up and sequenced. Nucleotides 2956 to 3336 was clearly interpreted. While we did not detect any mutation in the sequence (nucleotides 2956 to 3336) of the TLW3 progeny, we detected mutations in most of the progenies of TLIle that had been propagated in tomato plants. Most of the clones of TLIleprogenies propagated in four plants had mutated Ile979 (AUU) to Asn (AAU) [E1-P1], and Ile979 (AUU) to Phe (UUU) [E1-P2, E2-P1 and E2-P5]. The single mutation alone could allow the mutant to replicate in tomato cells (Hamamoto et al. 1997; H. Hamamoto, Y. Watanabe, H. Kamada, and Y. Okada, unpublished results). These results suggest that TLIle might have replicated at a very low level in tomato cells, and a virus that had acquired the mutation was selected for and became dominant in tomato plants. Two clones (one from E1-P2 and the other from E2-P1), however, retained Ile979. These clones had a second-site mutation [Glu982 (GAA) to Gly (GGA) and Asn978 (AAU) to Ser (AGU), respectively]. In E2-P2 tomato plant, all the eight clones retained Ile979 and two of them had second-site mutations at different positions in the region sequenced. One clone had three mutations, Val995 (GUU) to Ala (GCU), Ser1051 (UCG) to Leu (UUG), and Leu1071 (CUA) to Pro (CCA), while another clone had mutated Ser1075 (UCA) to Pro (CCA). The clones in E2-P2 plant might have second-site mutation(s) outside the region encompassed by nucleotides 2956 and 3336, which might have enabled the virus to replicate in tomato. However, the data here do not allow one to distinguish between either a very low level of replication of TLIle, or whether some of the secondsite suppressor mutants were generated during $\mathrm{T} 7$ polymerase transcription of TLIle. Thus, we did not pursue more sequencing analysis of these clones.

Since tobacco and tomato are known as common hosts for tobamoviruses (Dawson 1992), it was surprising to us that just a single amino acid substitution rendered the virus virtually incapable of replicating in tomato cells, while it had no effect on the ability to replicate in tobacco cells. We inoculated two other tomato cultivars, cv. Sekaiichi $[+/+]$ and cv. Sugarlamp $[+/+]$, with TLIle and delayed infection was also observed in them (data not shown). We have also inoculated protoplasts of cv. Craigella GCR 237 tomato cells [Tm-1/Tm-1] with TLIle by electroporation. No accumulation of RNA and coat protein was observed (data not shown). Thus, the phenomenon was not specific to cv. Craigella GCR 26. Since TLIle and previously constructed mutants with other substitutions at amino acid position 979 (Hamamoto et al. 1997) replicate in tobacco cells at levels similar to wild-type ToMV, substitutions at position 979 do not seem to affect the basic function of the 130and $180-\mathrm{kDa}$ proteins. It has been suggested that amino acid 979 plays a key role in the interaction between the 130- and $180-\mathrm{kDa}$ proteins and Tm-1 (Meshi et al. 1988). From the results of the present work, it seems that there might also be a factor that interacts with the 130 - and $180-\mathrm{kDa}$ proteins in the same manner as Tm-1 in tomato cells without $T m-1$ gene. In the process of replication of ToMV, a replication complex consisting of $130-$ and $180-\mathrm{kDa}$ proteins and some hostencoded factor(s) might be formed and act as a viral replicase (Ishikawa et al. 1993; Osman and Buck 1996). We suggest the following mechanism to explain how the Gln979 to Ile substitution alters host specificity. Amino acid 979 might play an essential role in the interaction between 130 - and $180-\mathrm{kDa}$ proteins and some host-encoded factor(s) that results in formation of the replication complex in tomato. The 130- and $180-\mathrm{kDa}$ proteins of TLIle might be unable to form a stable complex with the host-factor protein of tomato because of the Gln979 to Ile substitution. The analogous host factor of tobacco might be more flexible or interact with other amino acids and might be able to form a complex with the 130- and $180-\mathrm{kDa}$ proteins of both TLW3 and TLIle. Thus, the Gln979 to Ile substitution did not affect viral replication in tobacco cells. As Dawson and Hilf (1992) suggested, Tm-1 might encode an altered form of the host factor of tomato, which cannot interact with the 130 - and $180-\mathrm{kDa}$ proteins of the wildtype ToMV but can interact with those of the mutant with the Gln979 to Glu substitution.

\section{LITERATURE CITED}

Dawson, W. O. 1992. Tobamovirus-plant interactions. Virology 186: 359-367.

Dawson, W. O., and Hilf, M. E. 1992. Host-range determinants of plant viruses. Annu. Rev. Plant Physiol. Plant Mol. Biol. 43:527-555.

Fraser, R. S. S. and Loughlin, S. A. R. 1980. Resistance to tobacco mosaic virus in tomato: Effects of the Tm-1 gene on virus multiplication. J. Gen. Virol. 48:87-96.

Hamamoto, H., Sugiyama, Y., Nakagawa, N., Hashida, E., Matsunaga, Y., Takemoto, S., Watanabe, Y., and Okada, Y. 1993. A new tobacco mosaic virus vector and its use for the systemic production of angiotensin-I-converting enzyme inhibitor in transgenic tobacco and tomato. Bio/Technology 11:930-932.

Hamamoto, H., Watanabe, Y., Kamada, H., and Okada, Y. 1997. Amino acid changes in the putative replicase of tomato mosaic tobamovirus that overcome resistance in Tm-1 tomato. J. Gen. Virol. 78:461-464.

Haseloff, J., Goelet, P., Zimmern, D., Ahlquist, P., Dasgupta., R., and Kaesberg, P. 1984. Striking similarities in amino acid sequence among nonstructural proteins encoded by RNA viruses that have dissimilar genomic organization. Proc. Natl. Acad. Sci. USA 81:4358-4362.

Ishikawa, M., Meshi, T., Motoyoshi, F., Takamatsu, N., and Okada, Y. 1986. In vitro mutagenesis of the putative replicase genes of tobacco mosaic virus. Nucleic Acids Res. 14:8291-8305.

Ishikawa, M., Naito, S., and Ohno, T. 1993. Effects of the tom1 mutation of Arabidopsis thaliana on the multiplication of tobacco mosaic virus RNA in protoplasts. J. Virol. 67:5328-5338.

Kamer, G., and Argos, P. 1984. Primary structural comparison of RNAdependent polymerases from plant, animal and bacterial viruses. $\mathrm{Nu}-$ cleic Acids Res. 12:7269-7282.

Kunkel, T. A. 1985. Rapid and efficient site-specific mutagenesis without phenotypic selection. Proc. Natl. Acad. Sci. USA 82:488-492.

Meshi, T., Ishikawa, M., Motoyoshi, F., Semba, K., and Okada, Y. 1986. In vitro transcription of infectious RNAs from full-length cDNAs of tobacco mosaic virus. Proc. Natl. Acad. Sci. USA 83:5043-5047.

Meshi, T., Motoyoshi, F., Adachi, A., Watanabe, Y., Takamatsu, N., and Okada, Y. 1988. Two concomitant base substitutions in the putative replicase genes of tobacco mosaic virus confer the ability to overcome the effects of tomato resistance gene, Tm-1. EMBO J. 7:1575-1581.

Motoyoshi, F., and Oshima, N. 1979. Standardization in inoculation procedure and effect of a resistance gene on infection of tomato protoplasts with tobacco mosaic virus RNA. J. Gen. Virol. 44:801-806.

Ohno, T., Aoyagi, M., Yamanashi, Y., Saito, H., Ikawa, S., Meshi, T., and Okada, Y. 1984. Nucleotide sequence of the tobacco mosaic virus (tomato strain) genome and comparison with the common strain genome. J. Biochem. (Tokyo) 96:1915-1923. 
Osman, T. A. M., and Buck, K. W. 1996. Complete replication in vitro of tobacco mosaic virus RNA by a template-dependent, membranebound RNA polymerase. J. Virol. 70:6227-6234.

Otsuki, Y., Takebe, I., Ohno, T., Fukuda, M., and Okada, Y. 1977. Reconstitution of tobacco mosaic virus rods occurs bidirectionally from an internal initiation region: Demonstration by electron microscopic serology. Proc. Natl. Acad. Sci. USA 74:1913-1917.

Pelham, J. 1972. Strain-genotype interaction of tobacco mosaic virus in tomato. Ann. Appl. Biol. 71:219-228.

Watanabe, Y., Kishibayashi, N., Motoyoshi, F., and Okada, Y. 1987a. Characterization of $\mathrm{Tm}-1$ gene action on replication of common isolates and a resistance-breaking isolate of TMV. Virology 161: 527-532.

Watanabe, Y., Meshi, T., and Okada, Y. 1987b. Infection of tobacco protoplasts with in vitro transcribed tobacco mosaic virus RNA using an improved electroporation method. FEBS Lett. 219:65-69. 